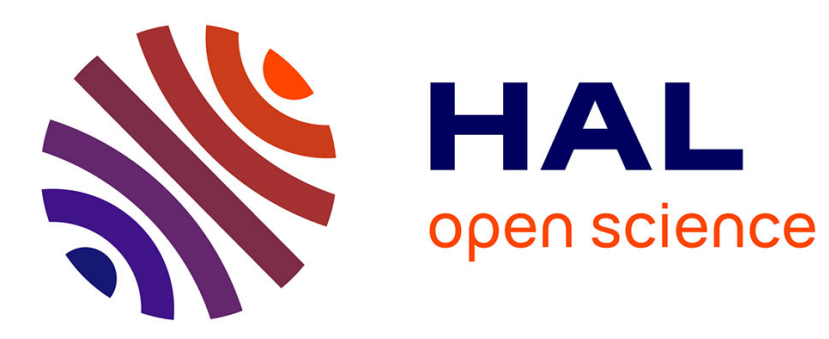

\title{
Spin Controlled Optical Radiation Pressure
}

Georgiy Tkachenko, Etienne Brasselet

\section{To cite this version:}

Georgiy Tkachenko, Etienne Brasselet. Spin Controlled Optical Radiation Pressure. Physical Review Letters, 2013, 111 (3), pp.033605. 10.1103/PhysRevLett.111.033605 . hal-00845806

\section{HAL Id: hal-00845806 https://hal.science/hal-00845806}

Submitted on 9 Mar 2018

HAL is a multi-disciplinary open access archive for the deposit and dissemination of scientific research documents, whether they are published or not. The documents may come from teaching and research institutions in France or abroad, or from public or private research centers.
L'archive ouverte pluridisciplinaire HAL, est destinée au dépôt et à la diffusion de documents scientifiques de niveau recherche, publiés ou non, émanant des établissements d'enseignement et de recherche français ou étrangers, des laboratoires publics ou privés. 


\title{
Spin Controlled Optical Radiation Pressure
}

\author{
Georgiy Tkachenko and Etienne Brasselet* \\ Université Bordeaux, Laboratoire Ondes et Matière d'Aquitaine, UMR 5798, F-33400 Talence, France \\ CNRS, Laboratoire Ondes et Matière d'Aquitaine, UMR 5798, F-33400 Talence, France
}

(Received 18 March 2013; published 17 July 2013)

\begin{abstract}
We report on the full control of the optical radiation pressure at fixed photon flux and incident angle by the photon spin. This is done by using transparent chiral liquid crystal droplets that enable a strong coupling between the linear and angular degrees of freedom of a light field. From these results, we anticipate optical sorting of particles with different chirality as well as novel optical trapping and micromanipulation strategies.
\end{abstract}

DOI: 10.1103/PhysRevLett.111.033605

PACS numbers: $42.50 . \mathrm{Wk}$

Optical radiation forces are the mechanical manifestation of the transfer of the linear momentum of light to matter, which basically occurs when light is reflected, refracted, scattered, or absorbed in the course of its propagation [1]. In particular, the discontinuity of the dielectric permittivity at the interface between two transparent, homogeneous, and dielectric media leads to optical radiation pressure that enables the displacement of solids $[2,3]$ or the deformation of fluid interfaces [4]. Here we report on the full control of the optical radiation pressure at fixed photon flux and incident angle by the photon spin. This is done by using transparent chiral liquid crystal droplets that enable a strong coupling between linear and angular degrees of freedom of light.

Chirality refers to the lack of mirror symmetry. A system whose mirror images cannot be superimposed by rotations and translations in space is said to be chiral. A basic example is our two hands, just like the Greek etymology (kheir) recalls. Quite naturally, two mirror images of a chiral entity are referred to as right- and left-handed versions of it. This concept holds for light as well, whose polarization handedness is said to be right or left depending on the sense of rotation of the electric field. Under appropriate conditions, the propagation of one of the two circular polarization states through a chiral optically anisotropic material may even be forbidden for a well-defined range of frequencies. A helical arrangement of the optical axis indeed combines dielectric periodic structuration, which leads to a Bragg photonic band gap, with chirality, which brings circular polarization sensitivity. A famous example is the circular Bragg reflection phenomenon in cholesteric liquid crystals [5], which we use here to experimentally demonstrate how mechanical effects driven by the radiation pressure of light can be fully controlled by the spin of photons. The principle of our experiment is sketched in Fig. 1.

The cholesteric mesophase refers to a chiral nematic state where the director $\mathbf{n}$ (a unit vector that defines the local averaged molecular orientation, $\mathbf{n}$ and $-\mathbf{n}$ being equivalent) twists in a well-defined direction with a helical pitch $p$ that is the distance over which $\mathbf{n}$ rotates by $2 \pi$, see upper sketch in Fig. 2(a). A planar cholesteric film having its helical axis along the normal to the film behaves as a perfect mirror for a normally incident collimated light beam with wavelength $\lambda_{0}$ inside the polarization photonic band gap, $\lambda_{-}<\lambda_{0}<\lambda_{+}$, centered on $\lambda_{B}$, see Fig. 2(a), and Bragg circular polarization state referred to as $\sigma_{B}$. In contrast, the orthogonally polarized beam referred to as anti-Bragg and labeled $\sigma_{A B}$ is transmitted through the film up to Fresnel reflection. At fixed incident wavelength $\lambda_{0}$, circular Bragg reflection thus occurs over a range of incidence angle around the normal incidence. This angular range for the external incidence angle is labeled $2 \theta_{B \text {,ext }}$, as illustrated in Fig. 1(b) in the case of a cholesteric droplet. That is to say, one can consider that total reflection occurs for the $\sigma_{B}$ polarization state when the external incidence angle satisfies $\theta_{\text {ext }}<\theta_{B, \text { ext }}=\arcsin \left[\left(n / n_{\text {ext }}\right) \sin \theta_{B}\right]$. In the latter expression, $n=\left(n_{\|}+n_{\perp}\right) / 2$ is the average refractive index of the cholesteric with $n_{\|, \perp}$ the refractive indices parallel and perpendicular to $\mathbf{n}$, and $n_{\text {ext }}\left(n_{\text {ext }}<n\right.$ in this work) is the refractive index of the medium in which the (a)

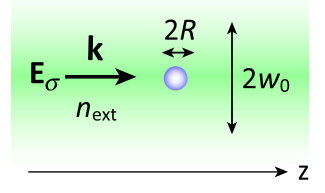

(b)

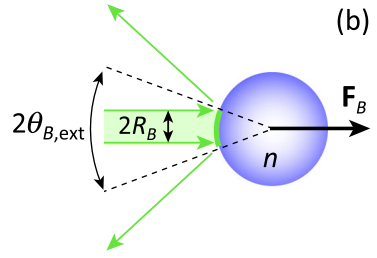

FIG. 1 (color online). (a) A radial cholesteric droplet of radius $R$ and average refractive index $n$ is immersed in a fluid with refractive index $n_{\text {ext }}$. The droplet is illuminated by on-axis circularly polarized Gaussian beam with waist $w_{0} \gg R$ (in practice $10<w_{0} / R<80$ ), vacuum wavelength $\lambda_{0}$, wave vector $\mathbf{k}$, and electric field $\mathbf{E}_{\sigma}$, where $\sigma= \pm 1$ refers to left- and righthanded circular polarization states. (b) Illustration of the optical radiation force $\mathbf{F}_{B}$ that results from the circular Bragg reflection phenomenon for the appropriate incident circular polarization state $\sigma_{B}$, for which the droplet behaves as a perfect mirror over an apex angle $2 \theta_{B \text {,ext }}$. 


\section{Planar film}

(a)
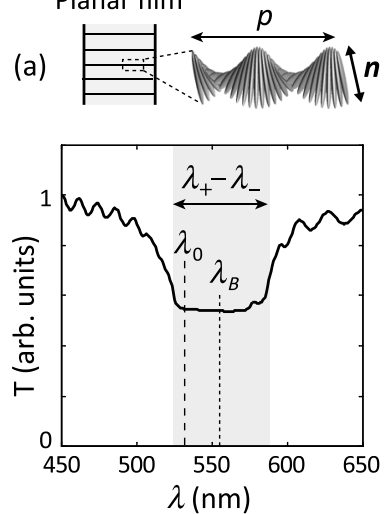

Radial droplet

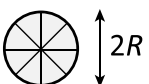

(b)

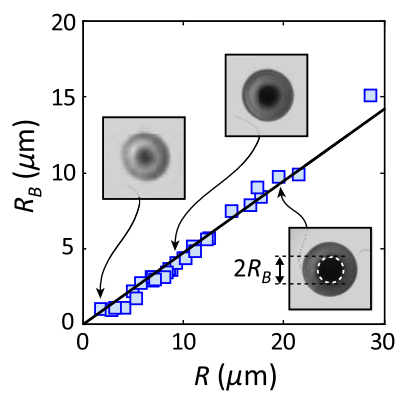

FIG. 2 (color online). (a) Unpolarized transmission spectrum of a right-handed cholesteric film with pitch $p$ and uniform alignment of helical axis along the normal to the film. The gray area refers to the spectral range of polarization selective Bragg photonic band gap $\lambda_{-}<\lambda<\lambda_{+}$with central wavelength $\lambda_{B}$. (b) In situ characterization of the reflecting cross-section area of spherical radial cholesteric droplets. Data show the radius $R_{B}$ of the zero-transmission area observed under uniform illumination at $\lambda_{0}=532 \mathrm{~nm}$ and Bragg incident circular polarization state $\sigma_{B}$ as a function of the droplet radius $R$. Solid line is the best linear fit.

cholesteric is immersed. In addition, accounting from $\lambda_{B}=n p$ and $\lambda_{+}-\lambda_{-}=p\left(n_{\|}-n_{\perp}\right)$ [6], one can show that $\theta_{B}=\arccos \left(\lambda_{0} / \lambda_{+}\right)$, at least in the limit of small $\theta_{B}$. A spherical cholesteric droplet with radial distribution of the helical axis, as sketched in Fig. 2(b), can therefore be considered as a spherical Bragg mirror with a total reflection cross-section area normalized to the geometrical crosssection area being equal to $\sin ^{2} \theta_{B \text {, ext }}$ for $\sigma_{B}$-polarized light and to zero for $\sigma_{A B}$-polarized light. This allows us to consider vivid experimental demonstration of spindependent optical radiation pressure by using free-floating droplets, as sketched in Fig. 1.

In our experiments, the cholesteric is right handed with pitch $p=347 \mathrm{~nm}$ (MDA-02-3211 from Merck) and $n_{\|}=$ 1.7013 and $n_{\perp}=1.5064$ at $589.3 \mathrm{~nm}$ wavelength and temperature $20^{\circ} \mathrm{C}$. From the transmission spectrum of a uniformly aligned film shown in Fig. 2(a), we measure the intrinsic Bragg angle $\theta_{B}=25.1^{\circ}$ at $\lambda_{0}=532 \mathrm{~nm}$. This is verified in situ for cholesteric droplets dispersed in glycerol, which ensures parallel anchoring of the director at cholesteric-glycerol interface, hence, a radial distribution of the helical axis for $p \ll R$ where $R$ is the droplet radius $[7,8]$. For this purpose, we measure the radius $R_{B}$ of the total reflection cross-section area under $\sigma_{B}$-polarized light at $\lambda_{0}=532 \mathrm{~nm}$, as a function of $R$, see Fig. 2(b) [9]. Since $R_{B}=R \sin \theta_{B \text {, ext }}$, we obtain $\theta_{B}=25.8^{\circ}$ from the best linear fit, in good agreement with the spectroscopic characterization.

In the first set of experiments, we follow the sketch shown in Fig. 1(a) and use spherical radial cholesteric droplets prepared by mechanical stirring at room
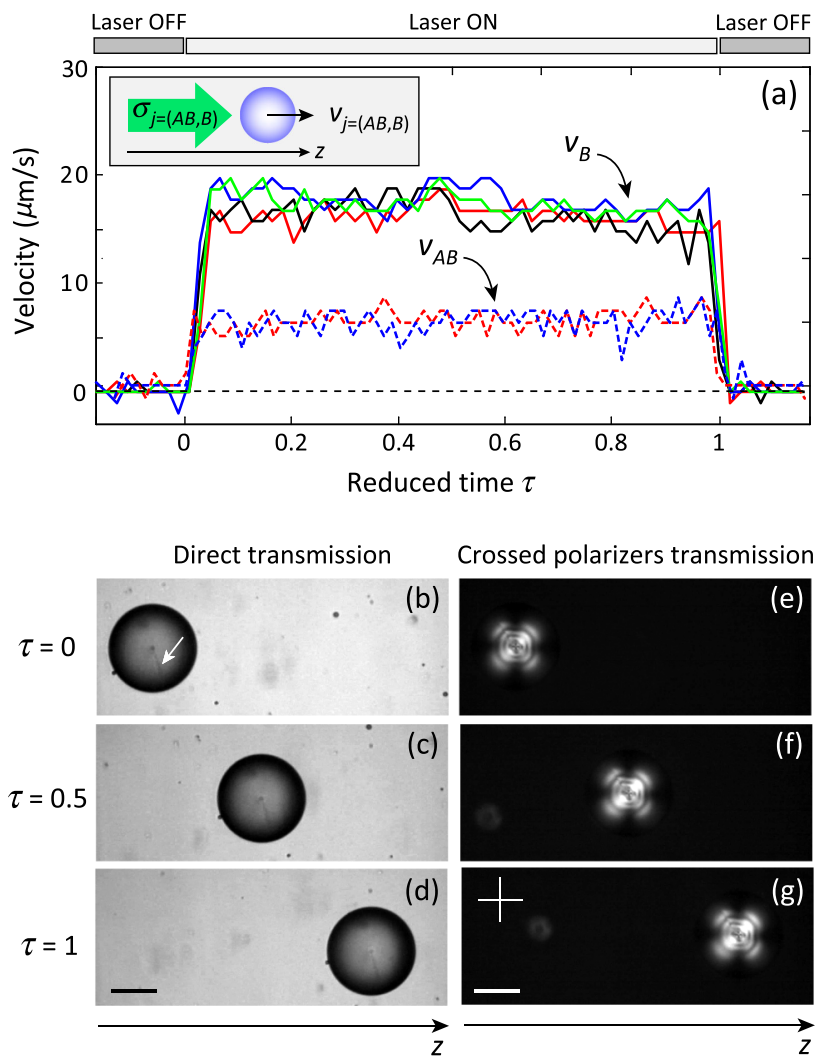

FIG. 3 (color online). One-beam experiment. (a) Droplet velocity dynamics as a function of reduced time $\tau=$ $\left(t-t_{\mathrm{on}}\right) /\left(t_{\mathrm{off}}-t_{\mathrm{on}}\right)$ for Bragg $\left(v_{B}\right.$, solid curves $)$ and antiBragg $\left(v_{A B}\right.$, dashed curves) laser beam illumination conditions, where $t_{\text {on }}$ and $t_{\text {off }}$ refer to switch on and off times of the laser beam, respectively. Different curves refer to independent experiments performed at power $P=970 \mathrm{~mW}$, waist $w_{0}=505 \mu \mathrm{m}$, and the droplet radius $R=28.4 \mu \mathrm{m}$. (b), (c), (d) Direct transmission snapshots of the droplet at times $\tau=0,0.5$, and 1 for the Bragg case. White arrow on panel (b) indicates the characteristic radial defect of the droplet. (e), (f), (g) Same as panels (b), (c), (d) for crossed polarizers transmission through the droplet, where the orientation of the polarizers is indicated on panel (g). Scale bar is $30 \mu \mathrm{m}$.

temperature of a small amount of cholesteric into an isodensity $25.2 \mathrm{wt} \%$ aqueous glycerol solution whose refractive index is 1.365 at $589.3 \mathrm{~nm}$ wavelength at $20^{\circ} \mathrm{C} \mathrm{[10]}$ and dynamic viscosity $\eta=2 \mathrm{mPas}$ [11]. This choice indeed eases observation of on-axis light-induced droplet displacement. Typical results are summarized in Fig. 3. Without laser illumination, the droplet is at rest. When the laser is turned on, the droplet almost immediately moves along the beam propagation direction with constant velocity. Partial control of the light-induced motion is obtained by setting the photon spin. The Bragg droplet velocity $v_{B}$ is indeed a few times larger than the anti-Bragg one, $v_{A B}$. Noteworthy, the radial structure of the droplet is unaltered during its displacement, as demonstrated by the optical transmission image sequences at the early, 
intermediate, and final stages of its laser-induced motion in the Bragg case, see Figs. 3(b)-3(d) (direct images) and Figs. 3(e)-3(g) (crossed polarized images). A characteristic radial defect $[7,8]$ is observed in the former case, see white arrow in Fig. 3(b), whereas the unchanged dark cross pattern indicates radially symmetric optical anisotropy in the latter case [8].

Such partial spin-controlled optomechanical effect in the one-beam experiment is actually explained by considering unavoidable axisymmetric angular redistribution of the optical linear momentum flux away from the propagation direction as the incident beam is refracted by the droplet, thereby leading to a net force directed along the $z$ axis. This interpretation is quantitatively tested by measuring the ratio $v_{A B} / v_{B}$ for various $R$, see square symbols in Fig. 4, and comparing it to the theoretical value predicted by the model described hereafter.

Rigorous treatment of light propagation in cholesteric films is not an easy task, though literature is available on this topic [12]. Nevertheless, this can be done numerically [13]. It becomes even trickier when considering droplets. In particular, scattering of light by a chiral particle is an issue that has started to be addressed only recently [14,15]. Moreover, Maxwell stress tensor formalism for inhomogeneous, chiral, and anisotropic optical media should be included as well as possible light-induced deformation of the shape of the droplet [16]. However, a simple but accurate physical picture of the problem can be handled from the following assumptions. (i) Since $p \ll R$ and $p \sim \lambda$, we describe light scattering in a ray-optics approach. (ii) Since $R \ll w_{0}$, the incident beam with power $P$ is considered as a plane wave with intensity $I_{0}=2 P /\left(\pi w_{0}^{2}\right)$. (iii) Since the optical radiation pressure $\left(\Pi_{R}\right)$ is much smaller than the Laplace pressure $\left(\Pi_{L}\right)$, we neglect droplet deformation. Indeed $\Pi_{R} \sim P /\left(w_{0}^{2} c\right) \sim 10^{-2} \mathrm{~Pa} \ll \Pi_{L} \sim \gamma / R \sim 10^{3} \mathrm{~Pa}$, with $c$ the speed of light in vacuum, $\gamma$ the surface tension

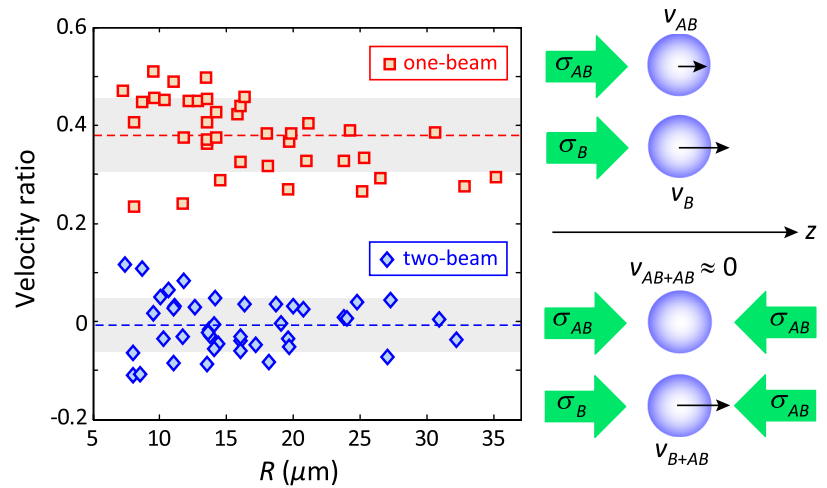

FIG. 4 (color online). Two-beam experiment. Experimental droplet velocity ratio $v_{A B} / v_{B}$ and $v_{A B+A B} / v_{B+A B}$ are plotted as a function of droplet radius $R$ in one- and two-beam experiments, respectively. Total power of each beam is $P=780 \mathrm{~mW}$. Dashed lines refer to mean values whereas gray areas correspond to standard deviation ranges. of the interface between cholesteric and host fluid, and typical radius $R=10 \mu \mathrm{m}$. (iv) Since the circular polarization photonic band gap has relatively sharp edges, see Fig. 2(a), a radial cholesteric droplet is considered as a perfectly reflecting mirror for incident angles $\theta<\theta_{B \text {,ext }}$ in the Bragg case and otherwise as a usual dielectric sphere of refractive index $n$ (i.e., when $\theta_{B \text {,ext }}<\theta<\pi / 2$ in the Bragg case and $0<\theta<\pi / 2$ in the anti-Bragg case). (v) Within the ray-optics description, the net force exerted on the cholesteric droplet is evaluated by calculating the net change of linear momentum of the light field as it interacts with the droplet. For this purpose, we attribute the Minkowski linear momentum $\hbar k$ per photon pointing along each geometrical ray, where $k$ is the wave number in the considered medium, and $\hbar$ is the reduced Planck's constant. Such a procedure has indeed been validated experimentally $[17,18]$ and demonstrated theoretically [19] for the problem of optical radiation pressure exerted on a mirror immersed in a dielectric fluid. (vi) Since the contribution of absorption of the droplet to the absolute relative change of the optical linear momentum along $z$ over the droplet cross-section area (referred to as $\epsilon$ ) is much smaller than the contribution produced by reflection and refraction, we consider the cholesteric as a transparent dielectric. Indeed $\epsilon_{\text {abs }} \sim \alpha_{\text {droplet }} R \sim 10^{-4} \ll \epsilon_{\text {refl, refr }} \sim 10^{-1}$ with typical absorption coefficient $\alpha_{\text {droplet }} \leqslant 10 \mathrm{~m}^{-1}$ and radius $R=$ $10 \mu \mathrm{m}$. (vii) Since light-induced bulk flow that results from the light absorption by the host fluid itself is typically 2 orders of magnitude smaller than observed droplet velocities in single-beam experiments, we consider the host fluid at rest. Indeed $v_{\text {fluid }} \sim \alpha_{\text {fluid }} P /(\eta c)$, see section 1.2.1 in Ref. [20] where $\alpha_{\text {fluid }}$ is the host fluid absorption coefficient. This gives $v_{\text {fluid }} \sim 0.1 \mu \mathrm{m} \mathrm{s}^{-1} \mathrm{~W}^{-1}$ with $\alpha_{\text {fluid }}=$ $0.035 \mathrm{~m}^{-1}$ at $25^{\circ} \mathrm{C}$ and $532 \mathrm{~nm}$ wavelength, which is the one of water [21]. Note that in two-beam experiments, absorption-induced bulk flow formally vanishes since the two beams are counterpropagating, coaxial, and have equal powers.

Following the above-mentioned assumptions, the projection along the beam propagation direction $z$ of the elementary optical force exerted by a single beam on the surface element $d S=R^{2} \sin \theta d \theta d \phi, \theta$ and $\phi$ being the polar and azimuthal angles in the spherical coordinate system centered on the droplet, is (see, for instance, Ref. [22])

$$
d F_{j}(\theta, \phi)=f_{j}(\theta) n_{\mathrm{ext}} \frac{I_{0}}{c} \cos \theta d S(\theta, \phi),
$$

with $j=(A B, B)$ and

$$
\begin{aligned}
f_{j}(\theta)=1 & +\mathcal{R}_{j}(\theta) \cos 2 \theta \\
& -\mathcal{T}_{j}^{2}(\theta) \frac{\cos \theta_{\text {out }}+\mathcal{R}_{j}(\theta) \cos 2 \theta}{1+2 \mathcal{R}_{j}(\theta) \cos 2 \theta_{\text {int }}+\mathcal{R}_{j}^{2}(\theta)},
\end{aligned}
$$

where $\mathcal{R}_{j}$ and $\mathcal{T}_{j}=1-\mathcal{R}_{j}$ are the reflectance and transmittance of the droplet interface, and $\theta_{\text {out }}=2\left(\theta-\theta_{\text {int }}\right)$ 
with $\theta_{\text {int }}=\arcsin \left[\left(n_{\text {ext }} / n\right) \sin \theta\right]$. Following the abovementioned assumption (iv), $\quad \mathcal{R}_{A B}(\theta)=\left[\mathcal{R}_{\|}(\theta)+\right.$ $\left.\mathcal{R}_{\perp}(\theta)\right] / 2$ for all $\theta$, where $\mathcal{R}_{\|}(\theta)=\left[\tan \left(\theta-\theta_{\text {int }}\right) / \tan (\theta+\right.$ $\left.\left.\theta_{\text {int }}\right)\right]^{2}$ and $\mathcal{R}_{\perp}(\theta)=\left[\sin \left(\theta-\theta_{\text {int }}\right) / \sin \left(\theta+\theta_{\text {int }}\right)\right]^{2}$ are the reflectances of plane waves polarized parallel and perpendicular to the incidence plane [23]. On the other hand, $\mathcal{R}_{B}\left(0<\theta<\theta_{B, \text { ext }}\right)=1 \quad$ and $\quad \mathcal{R}_{B}\left(\theta_{B, \text { ext }}<\theta<\pi / 2\right)=$ $\mathcal{R}_{A B}(\theta)$.

In the one-beam situation, the integration of Eq. (1) over the hemisphere gives the net force $F_{j}$. Then, in the limit of small Reynolds number as is the present case, the steady state droplet velocity $v_{j}$ is estimated from the balance between the viscous force of magnitude $6 \pi \eta R v_{j}$ exerted by the surrounding fluid on the moving droplet and the optical force $F_{j}$. The expression for the viscous force accounts for the fact that the viscosity of the surrounding fluid is much smaller than the effective viscosity of the cholesteric droplet, see [24]. We obtain

$$
v_{j}=F_{j} /(6 \pi \eta R),
$$

from which we can readily derive the ratio $v_{A B} / v_{B}$. As shown in Fig. 4, we find the experimental value $v_{A B} / v_{B}=$ $0.38 \pm 0.075$. From our model, by using $\theta_{B \text {,ext }}$ as the only adjustable parameter, we obtain an effective Bragg angle $\theta_{B}=15.3^{\circ}\left(+3.1^{\circ} /-2.3^{\circ}\right)$. This value is smaller than the ones measured from spectroscopic [Fig. 1(a)] and direct imaging [Fig. 1(b)] measurements. However, this is actually expected recalling that our model assumes perfect Bragg reflection over the effective Bragg angular band gap. We thus conclude to a fair description of our observations, thereby validating that (i) the physical picture is properly grasped, and (ii) the scattering of light by the droplet prevents full control of the optical radiation pressure by the photon spin.

In the second set of experiments, we overcome the above-mentioned fundamental limitation by using two coaxial, collimated, circularly polarized, and counterpropagating beams with equal powers and waists, one being either $\sigma_{B}$ or $\sigma_{A B}$ polarized whereas the other is $\sigma_{A B}$ polarized. When both beams are $\sigma_{A B}$ polarized, the individual contributions of the two beams to the total optical scattering force indeed perfectly cancel each other. A nonzero net force is exerted on the droplet otherwise. That is to say, $v_{A B+A B}=0$ and $v_{B+A B} \neq 0$ are expected, the former case being confirmed experimentally whatever the droplet radius, see diamond symbols in Fig. 4. We indeed measure $v_{A B+A B} / v_{B+A B}=-0.0083 \pm 0.055$, hence, achieving full control of the optical radiation pressure exerted on a microscopic object by a mere change of the photon spin.

The dependence on $P$ and $R$ of the droplet velocity in the "Bragg or anti-Bragg" case is shown in Fig. 5. In this figure, the inverse of the characteristic time of the droplet motion $v_{B+A B} / R$ is shown as a function of total power $P$ of one beam for various droplets. To confront these observations with our model, we use Eq. (2) with

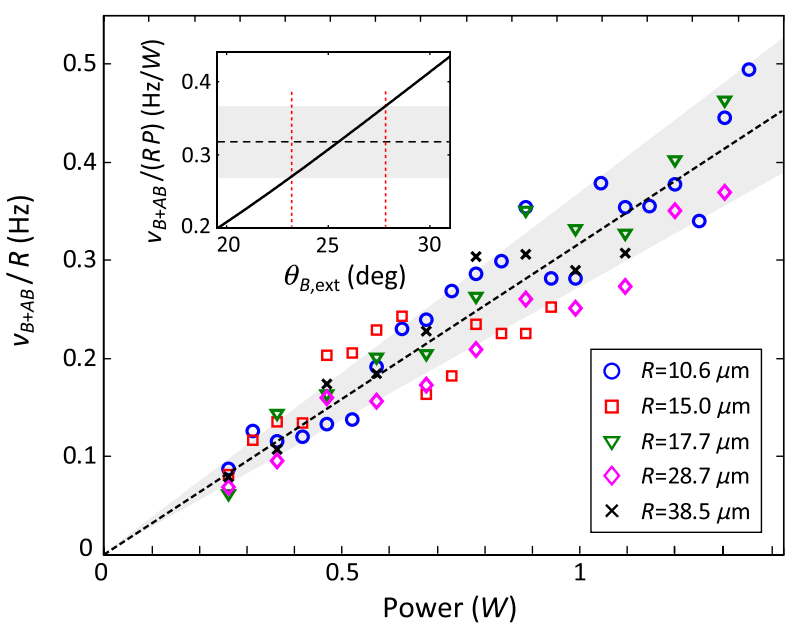

FIG. 5 (color online). The ratio $v_{B+A B} / R$ is plotted as a function of total beam power $P$ of one beam for various values of the droplet radius $R$. Dashed curve refers to the best linear fit from Eq. (3). Inset: solid line is the theoretical ratio $v_{B+A B} /(R P)$ vs $\theta_{B \text {,ext }}$ whereas the dashed line and gray area refer to the mean value and the standard deviation range of $v_{B+A B} /(R P)$, respectively. This allows us to deduce the precision of $\theta_{B}$, see vertical dotted lines.

$F_{B+A B}=F_{B}-F_{A B}$ and $F_{A B+A B}=0$ as the total force exerted on the droplet in the "Bragg and anti-Bragg" and "anti-Bragg and anti-Bragg" cases, respectively.

Since the refractive index contrast between the droplet and the host fluid is moderate, $n / n_{\mathrm{ext}}=1.17$, and that $\theta_{B \text {,ext }} \sim 20^{\circ}-30^{\circ}$, the Fresnel contributions to $F_{B+A B}$ can be approximated (up to a few percent) assuming $\mathcal{R}_{A B} \rightarrow 0$ and $\theta_{\text {out }} \rightarrow 0$. This leads to

$$
\frac{v_{B+A B}}{R}=\frac{n_{\mathrm{ext}}}{3 \pi \eta w_{0}^{2} c}\left(1-\cos ^{4} \theta_{B, \mathrm{ext}}\right) P .
$$

Following Eq. (3), the best linear fit using $\theta_{B \text {,ext }}$ as the only adjustable parameter (see dashed line in Fig. 5) gives $\theta_{B}=$ $21.5^{\circ}\left(+1.9^{\circ} /-1.9^{\circ}\right)$. As emphasized by Eq. (3), a quantitative analysis in the two-beam experiments is more difficult than in the one-beam case since the fitting procedure involves additional parameters, namely, $\eta$ and $w_{0}$. The beam waist is measured from direct imaging whereas the viscosity is taken from tabulated data $(\eta=2 \mathrm{mPas})$ without measuring it for our actual aqueous glycerol solution. This could explain the difference of $\simeq 6^{\circ}$ between the values of $\theta_{B}$ obtained from one-beam and two-beam experiments. We conclude that our model gives an overall satisfying description of observations in the two-beam experiments as well.

We have presented a quantitative experimental study demonstrating that optical radiation pressure can be fully controlled by the spin angular momentum of light, at fixed photon flux and incidence angle, in contrast to existing optical micromanipulation techniques, though we noticed that optical tweezers endowed with spin-controlled optical 
gradient forces have been recently reported using cholesteric droplets [25]. A straightforward anticipated application is the development of chiral optical sorting, which would bring enhanced functionality to optical chromatography [26]. This allows us to envision applications for the pharmaceutical industry related to the ability to sort materials with different chiralities. Nanoactuation driven by optical radiation pressure [27] is another topic that may benefit from such a polarization control, as discussed in the few dedicated theoretical studies reported so far [14,28]. Finally, at a larger spatial scale, our findings emphasized the spin angular momentum of light as a novel ingredient to drive solar sails [29].

We are grateful to N. Katsonis for providing the cholesteric material and its spectroscopic characterization.

*e.brasselet@loma.u-bordeaux1.fr

[1] A. Jonas and P. Zemanek, Electrophoresis 29, 4813 (2008).

[2] P. Lebedew, Ann. Phys. (Berlin) 311, 433 (1901).

[3] E. F. Nichols and G. F. Hull, Phys. Rev. 13, 307 (1901).

[4] A. Ashkin and J. M. Dziedzic, Phys. Rev. Lett. 30, 139 (1973).

[5] D. W. Berreman and T. L. Scheffer, Phys. Rev. Lett. 25, 577 (1970).

[6] P. Oswald and P. Pieranski, Nematic and Cholesteric Liquid Crystals: Concepts and Physical Properties Illustrated by Experiments (Taylor and Francis/CRC Press, London, 2005).

[7] J. Bezic and S. Zumer, Liq. Cryst. 11, 593 (1992).

[8] F. Xu and P. P. Crooker, Phys. Rev. E 56, 6853 (1997).

[9] In practice, once a droplet has been selected, its radius $R$ is measured by data analysis of the recorded image. The analysis protocol consists in an azimuthal averaging of the image intensity distribution by fixing the origin as the center of the droplet and differentiating the obtained intensity profile. Then, $R$ is easily and precisely determined by locating the corresponding inflexion point. The Bragg radius $R_{B}$ is determined in the same way.

[10] Glycerine Producers' Association, Physical Properties of Glycerine and Its Solutions (Glycerine Producers' Association, New York, 1963), p. 13.

[11] N. S. Cheng, Ind. Eng. Chem. Res. 47, 3285 (2008).

[12] V.A. Belyakov, V.E. Dmitrienko, and V.P. Orlov, Sov. Phys. Usp. 22, 64 (1979).

[13] Q. Hong, T.X. Wu, and S. T. Wu, Liq. Cryst. 30, 367 (2003).

[14] D. V. Guzatov and V. V. Klimov, Quantum Electron. 41, 526 (2011).

[15] Z.-S. Wu, Q.-C. Shang, and Z.-J. Li, Appl. Opt. 51, 6661 (2012).

[16] J. Z. Zhang and R. K. Chang, Opt. Lett. 13, 916 (1988).

[17] R. V. Jones and J. C. S. Richards, Proc. R. Soc. A 221, 480 (1954).

[18] R. V. Jones and B. Leslie, Proc. R. Soc. A 360, 347 (1978).

[19] B. A. Kemp and T. M. Grzegorczyk, Opt. Lett. 36, 493 (2011).

[20] R. Wunenburger, B. Issenmann, E. Brasselet, C. Loussert, V. Hourtane, and J.-P. Delville, J. Fluid Mech. 666, 273 (2011).

[21] G. M. Hale and M. R. Querry, Appl. Opt. 12, 555 (1973).

[22] G. Roosen and C. Imbert, Phys. Lett. 59A, 6 (1976).

[23] M. Born and E. Wolf, Principles of Optics (Pergamon, New York, 2005).

[24] Z.-G. Feng, E. E. Michaelides, and S. Mao, Fluid Dyn. Res. 44, 025502 (2012).

[25] G. Cipparrone, A. Mazzulla, A. Pane, R. J. Hernandez, and R. Bartolino, Adv. Mater. 23, 5773 (2011).

[26] T. Imasaka, Y. Kawabata, T. Kaneta, and Y. lshidru, Anal. Chem. 67, 1763 (1995).

[27] V. Petrov, J. Hahn, J. Petter, M. Petrov, and T. Tschudi, Opt. Lett. 30, 3138 (2005).

[28] B. M. Ross and A. Lakhtakia, Optik (Stuttgart) 119, 7 (2008).

[29] G. A. Swartzlander, T. J. Peterson, A. B. Artusio-Glimpse, and A. D. Raisanen, Nat. Photonics 5, 48 (2010). 